\title{
A Modal View of the Semantics of Theoretical Sentences
}

\author{
Holger Andreas \\ Institute for Philosophy, Logic, and Theory of Science - LMU Munich
}

May 3, 2009

\begin{abstract}
Modal logic has been applied in many different areas, as reasoning about time, knowledge and belief, necessity and possibility, to mention only some examples. In the present paper, an attempt is made to use modal logic to account for the semantics of theoretical sentences in scientific language. Theoretical sentences have been studied extensively since the work of Ramsey and Carnap. The present attempt at a modal analysis is motivated by there being several intended interpretations of the theoretical terms once these terms are introduced through the axioms of a theory.
\end{abstract}

Keywords: Theoretical terms, modal logic, Ramsey sentence.

\section{Introduction}

A theoretical term is a non-logical symbol whose meaning is introduced through axioms of a theory which - at least prima facie - do not count as definitions. One of the few attempts at a characterization of theoretical terms by formal means was made by Ramsey in his (1950). Another way of accounting for the semantic peculiarities of theoretical terms will be expounded in the present paper. This account is based on Carnap's notion of an indirect interpretation of theoretical terms by postulates. It is akin to the Ramsey view but, at the same time, apt to overcome certain difficulties inherent in this view when it comes to the formal representation of the claim that a theoretical sentence is true.

What is the connection between theoretical terms and modal logic? The truthrules for theoretical sentences, as they will be expounded here, show a striking similarity to the rules for the box operator in modal logic. For this reason, we are speaking of a modal view of the semantics of theoretical sentences. An explicit modal notation for the assertion of theoretical sentences in this view 
will be given. It will also be shown that the modal view represents deductive reasoning with theoretical sentences more properly than the Ramsey view does.

Following upon a representation of the Ramsey view in section 2 is the exposition of the modal view in section 3. In section 4, two notations for the assertion of a theoretical sentence are introduced. These notations can be seen to represent the semantics of such sentences. Deductive reasoning with theoretical sentences is addressed in section 5 . In section 6 , we deal with competing theories, showing that the modal view allows for a theory-immanent notion of truth.

\section{The Ramsey View}

For an outline of the Ramsey view of scientific theories, some notational conventions need to be introduced. These conventions will also be used in the remainder of the paper. Essential to Ramsey's and other accounts of scientific language is the division of non-logical symbols into a set $V_{o}$ of observational terms and another set $V_{t}$ of theoretical terms. A theory $T C$ may be formulated in a language $L\left(V_{o}, V_{t}\right)$. Both the observational language $L\left(V_{o}\right)$ and the theoretical language $L\left(V_{o}, V_{t}\right)$, which contains $L\left(V_{o}\right)$ as a fragment, are assumed to be of first order. The symbol $T C$ stands for a conjunction of axioms, where the T-axioms contain only $V_{t}$ terms as non-logical symbols, while the C-axioms contain both $V_{o}$ and $V_{t}$ terms. We will use $\Phi_{T C}$ to refer to the set of T- and Caxioms. A theory may be given either as the conjunction of its $\mathrm{T}$ - and $\mathrm{C}$-axioms or as the set of these axioms.

The Ramsey sentence of a theory $T C$ in the language $L\left(V_{o}, V_{t}\right)$ is obtained by the following transformations of the conjunction of $\mathrm{T}$ - and $\mathrm{C}$-axioms. First, replace the theoretical terms in this conjunction by appropriate second order variables. Then, bind these variables by second order existential quantifiers. As result one obtains a second order sentence of the following form:

$\left(T C^{R}\right) \quad \exists X_{1} \ldots \exists X_{n} T C\left(n_{1}, \ldots, n_{k}, X_{1}, \ldots, X_{n}\right)$

where $X_{1}, \ldots, X_{n}$ are second order variables and $n_{1}, \ldots, n_{k}$ non-logical symbols of $L\left(V_{o}\right)$.

$T C^{R}$ is also called the Ramsey sentence of a theory $T C$. If one thinks that $T C^{R}$ should be preferred to $T C$ as a proposition of the theory $T C$, one holds the Ramsey view of scientific theories. This view differs from the standard one with regard to whether the axioms $\Phi_{T C}$ are considered individually meaningful statements. This is affirmed in the standard view but denied in the Ramsey view.

Of Ramsey's original exposition it seems justified to say that the second order variables are ranging over sets of individuals of the observational domain $D_{o}$. This assumption is not inherent in the Ramsey view as we may also assume that there is a domain $D_{t}$ of theoretical entities and that it is, first, sets of n-tuples of 
individuals of $D_{t}$ and, second, sets of n-tuples of individuals of both $D_{t}$ and $D_{o}$ that the second order variables are ranging over. The latter option was pursued by Carnap (1958)/(1975) and was recently taken in an account of the subject matter by Ketland (2004). It is also adopted in the present investigation. It is assumed, furthermore, that $V_{t}$ contains either no individual constants or only mathematical ones whose interpretation does not vary. ${ }^{1}$ In any case, there are no individual constants being subject to the method of existential quantification through which the Ramsey sentence is obtained.

Why should one prefer the Ramsey view to the standard one? In what follows, we will discuss two interrelated reasons for such a preference. One of these concerns the question of whether atomic theoretical sentences are individually meaningful, and the other an epistemological problem inherent in the standard view.

In the Ramsey view, it is denied that an atomic theoretical sentence is individually meaningful. For, once the theoretical terms are replaced by appropriate second order variables, we lack the expressive resources to use such a sentence for the purpose of making an assertion. Rather, the assertion of a theoretical sentence $\phi$ has to be expressed by putting $\phi$ into the scope of the quantifiers of the Ramsey sentence. More precisely, Ramsey seems to give the following instruction of how to translate a theoretical sentence $\phi$ in the standard view into a corresponding sentence in the Ramsey view:

$$
\begin{aligned}
& \exists X_{1} \ldots \exists X_{n}\left(T C ( n _ { 1 } , \ldots , n _ { k } , X _ { 1 } , \ldots , X _ { n } ) \wedge \phi _ { a } \left(\left[X_{1} / t_{1}\right], \ldots,\right.\right. \\
& \left.\left.\left[X_{n} / t_{n}\right]\right) \wedge \phi\left(\left[X_{1} / t_{1}\right], \ldots,\left[X_{n} / t_{n}\right]\right)\right)
\end{aligned}
$$

where $\phi_{a}$ stands for the conjunction of sentences which are affirmed already in the language, in addition to the axioms of $T C$, and $\phi_{a}\left(\left[X_{1} / t_{1}\right], \ldots,\left[X_{n} / t_{n}\right]\right)$ for the formula that is obtained by replacing every occurrence of a theoretical term in $\phi_{a}$ with its corresponding second order variable. Likewise, $\phi\left(\left[X_{1} / t_{1}\right], \ldots\right.$, $\left.\left[X_{n} / t_{n}\right]\right)$ is obtained through this procedure of replacing theoretical terms with second order variables. (See Ramsey (1950), p. 231n.) Henceforth, we shall write $\left(T C \wedge \phi_{a} \wedge \phi\right)^{R}$ to refer to a sentence of the form (1).

It is nevertheless questionable whether $\left(T C \wedge \phi_{a} \wedge \phi\right)^{R}$ is an appropriate representation of the meaning of $\phi$. For, as Ramsey points out, it may well be the case that $\left(T C \wedge \phi_{a} \wedge \phi\right)^{R}$ is true, while $\left(T C \wedge \phi_{a} \wedge \neg \phi\right)^{R}$ is also true. In this case, $\phi$ and $\neg \phi$ would have to be considered true in the Ramsey view. To avoid this, Ramsey says that a theoretical sentence $\phi$ means the difference between $\left(T C \wedge \phi_{a} \wedge \phi\right)^{R}$ and $\left(T C \wedge \phi_{a}\right)^{R}{ }^{2}$ It is not quite clear whether Ramsey himself distinguished between the meaning of a theoretical sentence and the meaning of an assertion of such a sentence. As we understand his line of thought, there is no difference. For, the meaning of a sentence is that kind of property we

\footnotetext{
${ }^{1}$ The latter assumption was made by Carnap (1958)/(1975).

2It should be noted that, in later discussions of Ramsification, $\phi_{a}$, the set of assertions that are made in the context of a given theory, is not taken into account. Nor will $\phi_{a}$ be considered to account for the meaning of theoretical sentences in the modal view to be developed in the present paper.
} 
are concerned with in understanding the assertion of a sentence. In section 4, we will analyze the formal means for representing the assertion of a theoretical sentence with the intent to explicate the meaning of such a sentence.

Ramsey's instruction of how to translate a theoretical sentence $\phi$ makes the meaning of such a sentence dependent on the axioms $\Phi_{T C}$ and on which other sentences are affirmed. The Ramsey view may therefore be seen as a first attempt to give a context theory of meaning in which the meaning of a theoretical sentence depends on the theoretical context in which it is being asserted. In this case, the meaning of a theoretical sentence is not solely compositionally determined by the meaning of the component expressions occurring in that sentence. The theoretical context is given by a scientific theory conferring meaning on scientific expressions and by theoretical assertions represented by $\phi_{a}$. Ramsey himself expresses the contextual dependencies of the meaning of theoretical sentences by figurative ways of speaking (Ramsey (1950), p. 231):

Any additions to the theory, whether in the form of new axioms or particular assertions like $\alpha(0,3)$, are to be made within the scope of the original $\alpha, \beta, \gamma$. They are not, therefore, strictly propositions by themselves just as the different sentences in a story beginning 'Once upon a time' have not complete meanings and so are not propositions by themselves.

It appears that Ramsey himself considered this point crucially important as his analysis of the contextual aspect of sentence meaning follows immediately upon his proposal to take $T C^{R}$ as the proposition of a theory in place of $T C$.

Let us now move to epistemological considerations lending support to the Ramsey view. Sneed (1979) discovered a serious problem inherent in the standard view but not arising in the Ramsey view. This problem concerns the mutual dependency of the extension of the axioms $\Phi_{T C}$ and the extension of the theoretical terms. On the one hand, we are to know the values of a theoretical term $t$ in order to find out whether the sentences of $\Phi_{T C}$ are true. On the other hand, it is simply impossible to determine the extension of $t$ without assuming the truth of some sentence of $\Phi_{T C}$ in advance. This epistemological circle has been termed the problem of theoretical terms by Sneed. Importantly, it does not arise in the Ramsey view. For, by $T C^{R}$ it is only claimed that there are extensions of the theoretical terms satisfying the postulates $\Phi_{T C}$ under a given interpretation of $L\left(V_{o}\right)$, but no claim is made by $T C^{R}$ whether or not the sentences of $\Phi_{T C}$ are true. For a thorough discussion see Sneed (1979), p. 30-40.

What Sneed has expounded as the problem of theoretical terms may show that the standard view leads to insurmountable epistemological difficulties so that agnosticism about scientific theories is inevitable under this view. Holistic and context theories of meaning have gained wide ranging acceptance among philosophers of science since Quine's (1961) and subsequent work as well as Feyerabend's (1962), but are expounded in many cases in a rather informal fashion. What is the relative merit of context theories of meaning in comparison to the standard semantics, in which every sentence is considered individually meaningful? To give a brief answer, context theories of meaning seem to accord much 
better with the manner we do grasp the meaning of scientific concepts than standard semantics does. Concepts, such as force or electromagnetic field, are introduced to the novice in the field through axioms of a scientific theory and thus explained in the context of such a theory. It is those axioms on which we rely on in measuring the quantities corresponding to scientific concepts. Hence, we can say that it is through these axioms that we grasp the meaning of scientific concepts. That our semantic theories may account for how speakers grasp the meaning of scientific concepts is a requirement on which Dummett (1991), p. 340, insisted with great emphasis. Of course, one is free to reject this requirement, but then it becomes questionable of what the explanatory value of our semantic theories consists in.

Upon this appraisal of the Ramsey view two critical notes may be sounded. First, it is hard to see how our logical practice of using a theoretical sentence $\phi$ in derivations accords with Ramsey's proposal to transform the assertion of such a sentence into the Ramsey view, that is, to assert $\left(T C \wedge \phi_{a} \wedge \phi\right)^{R}$ in place of $\phi$. The notation suggested by Ramsey is obviously not the one used in science. Adopting it would lead to highly complex expressions that seem unsuitable for our inferential practice. ${ }^{3}$ Second, Ramsey's proposal for accounting for the meaning of a theoretical sentence $\phi$ cannot be seen as a formal representation as it refers merely to the difference between two formal notations, i. e., to the difference between $\left(T C \wedge \phi_{a} \wedge \phi\right)^{R}$ and $\left(T C \wedge \phi_{a}\right)^{R}$. Our aim in the next two sections will be to give a model-theoretic notation for asserting theoretical sentences that has the merits of the Ramsey view, as just explained, but allows a much simpler notation for the assertion of theoretical sentences.

\section{A Modal View}

As indicated above, the sentences that bestow meaning on a theoretical term by way of an interpretation do - at least prima facie - not qualify as definitions in the strict sense the term definition is understood in mathematical logic. This is what makes explanation of the interpretation of theoretical terms difficult. It is tempting to think that the Ramsey view is the only way of accounting for the semantics of theoretical terms. Another view shall, however, be expounded in this section. This view draws heavily on Carnap's Logical Syntax of Language (1937) as well as his Foundations of Logic and Mathematics (1939). What follows is a condensed exposition of an account that has been developed elsewhere in greater detail. (See Andreas (2007), p. 97-164.)

\footnotetext{
${ }^{3}$ Arguably, this problem can be solved by taking $T C^{R}$ as the empirical claim of the theory and $T C^{R} \rightarrow T C$ as the single postulate conferring meaning on the theoretical terms. Then, $T C$ can be inferred from these two sentences. This proposal was made by Carnap in his (1958)/(1975). It should be noted, however, that the proposal is motivated by a semantic explanation of how to understand the postulate $T C^{R} \rightarrow T C$, which is given in an informal fashion and not covered by Carnap's technical work on predicate logic. Even though the modal view presented in the next section is designed to apply to the postulates $\Phi_{T C}$, it is also applicable to the single postulate $T C^{R} \rightarrow T C$. It may therefore be seen as clarifying Carnap's informal explanation of how the interpretation of theoretical terms can be determined by this postulate.
} 
In his (1939), p. 65-69, Carnap introduced the notion of an indirect interpretation of a non-logical symbol as follows. This notion is to be understood against the background of a direct interpretation. The interpretation of a non-logical symbol is direct if it consists in an assignment of an extension or an intension to that symbol and is given by an expression of the meta-language. By contrast, the interpretation of a non-logical symbol is indirect if it is given by one or several sentences of the object language which have the status of being non-logical axioms in the calculus. Carnap's explanation of the logical status of postulates mainly concerns their syntactic function: Postulates are, according to Carnap, non-logical axioms which are taken to be valid in the calculus and which therefore can be used in every derivation, as is the case with a definition.

Let us now, in addition to Carnap's syntactic explanation, move the focus onto the semantic function of postulates. We propose to adopt the following explanation:

Explanation 1. A set $\Phi_{T C}$ of postulates that interprets a set $V_{t}$ of theoretical terms on the basis of a language $L\left(V_{o}\right)$ imposes a constraint on the intended interpretations of the language $L\left(V_{o}, V_{t}\right)$. This means, in terms of model-theoretic semantics, that every admissible $L\left(V_{o}, V_{t}\right)$ structure must satisfy $\Phi_{T C}$. The admissible $L\left(V_{o}, V_{t}\right)$ structures may have two domains of interpretation, one observational domain $D_{o}$ and a domain of theoretical entities $D_{t}$. With respect to a given $L\left(V_{o}\right)$ structure $\mathfrak{A}$, there may be several $L\left(V_{o}, V_{t}\right)$ structures that extend $\mathfrak{A}$ and satisfy $\Phi_{T C}$.

This explanation can be motivated by an analysis of the similarities and dissimilarities between the concept of a definition and the concept of a postulate (Andreas (2007)). It is important to note that we cannot expect the interpretation of a symbol through postulates to result in a unique determination of the extension of this symbol. This contrasts sharply with the interpretation of a symbol through a definition. In the case of a definition, the uniqueness of interpretation is an implication of Beth's definability theorem. (For discussion of this point see also van Benthem (1978), p. 332.)

Why does the interpretation of theoretical terms through postulates not result in a unique determination of the extension of these terms? One can verify the proposition of non-uniqueness for several quantities in physics, as mass, force, temperature, or the intensity of the electromagnetic field. Further, Ramsey (1950) and Braithwaite (1955) tried to show that certain attempts to define theoretical terms would block the growth of the respective scientific theory. That the extension of theoretical terms is not uniquely determined is also implicit in Ramsey's claim that both $\left(T C \wedge \Phi_{a} \wedge \phi\right)^{R}$ and $\left(T C \wedge \Phi_{a} \wedge \neg \phi\right)^{R}$ may well be true. Finally, it can be shown that the proposition of non-uniqueness concerning the interpretation of theoretical terms is an implication of Carnap's doctrine of partial interpretation (Andreas (2007), p. 157). In retrospect, we can say that the Ramsey view has the merit of not requiring the extension of theoretical terms to be uniquely determined. ${ }^{4}$

\footnotetext{
${ }^{4}$ David Lewis attempted to define theoretical terms by means of a definite description in his (1970). For a critical discussion of this attempt see Schurz (2005). Schurz, convincingly,
} 
In Carnap's so-called dual-level conception of scientific language, on which we are aiming to elaborate, the interpretation of the observational language is assumed to be determined uniquely by rules of designation reflecting the intended interpretation of $L\left(V_{o}\right)$. There is thus a twofold constraint on the intended interpretations of $L\left(V_{o}, V_{t}\right)$, one by the interpretation of $L\left(V_{o}\right)$ and another by the postulates $\Phi_{T C}$. In other words, a $L\left(V_{o}, V_{t}\right)$ structure is admissible iff it satisfies the postulates and extends the intended interpretation of $L\left(V_{o}\right)$ to include an interpretation of the theoretical terms $V_{t}$.

From such an understanding of an admissible structure, truth rules for theoretical sentences may be set up as follows. Let $\mathfrak{A}_{o}$ designate the intended interpretation of the observational language. ${ }^{5}$ Let $\operatorname{MOD}\left(\Phi_{T C}\right)$ designate the set of $L\left(V_{o}, V_{t}\right)$ structures that satisfy the postulates $\Phi_{T C}$ and $\operatorname{EXT}\left(\mathfrak{A}_{o}, V_{t}\right.$, $\left.D_{t}\right)$ the $L\left(V_{o}, V_{t}\right)$ structures that extend $\mathfrak{A}_{o}$ to interpret the $V_{t}$ symbols, where these symbols are allowed to have argument positions that are interpreted in $D_{t}$. More precisely, a $L\left(V_{o}, V_{t}\right)$ structure is a quadruple $\left(D_{o}, D_{t}, \mathfrak{a}_{o}, \mathfrak{a}_{t}\right)$, where $D_{o}$ designates the domain of interpretation of observational terms, $\mathfrak{a}_{o}$ the interpretation function for observational terms, and $\mathfrak{a}_{t}$ the interpretation function for theoretical terms. The latter kind of terms have at least one argument position that is interpreted in $D_{t}$. Every extension of a given $L\left(V_{o}\right)$ structure $\mathfrak{A}$ must, by definition, agree with $\mathfrak{A}$ on the interpretation of the $V_{o}$ symbols. A theoretical sentence is one in which at least one theoretical term occurs. Then, the truth of such a sentence is defined by the following definitions:

Definition 1. $\mathbf{S}_{\mathfrak{a}}$ designates the set of $L\left(V_{o}, V_{t}\right)$ structures that are admissible under an interpretation of the $V_{t}$ symbols by the postulates $\Phi_{T C}$. It is defined as follows:

$$
\mathbf{S}_{\mathfrak{a}}:=\left\{\begin{array}{cc}
\operatorname{MOD}\left(\Phi_{T C}\right) \cap \operatorname{EXT}\left(\mathfrak{A}_{o}, V_{t}, D_{t}\right) & \text { if } \operatorname{MOD}\left(\Phi_{T C}\right) \cap \operatorname{EXT}\left(\mathfrak{A}_{o}, V_{t}, D_{t}\right) \neq \emptyset, \\
\operatorname{EXT}\left(\mathfrak{A}_{o}, V_{t}, D_{t}\right) & \text { if } \operatorname{MOD}\left(\Phi_{T C}\right) \cap \operatorname{EXT}\left(\mathfrak{A}_{o}, V_{t}, D_{t}\right)=\emptyset .
\end{array}\right.
$$

Definition 2. $\nu(\phi)$ is the function that assigns truth-values to theoretical sentences of $L\left(V_{o}, V_{t}\right)$. It is defined as follows:

i) $\nu(\phi):=T$ iff for every structure $\mathfrak{A} \in \mathbf{S}_{\mathfrak{a}}, \mathfrak{A}=\phi$ holds

argues that Lewis's proposal only works on the assumption that the second order variables of the Ramsey sentence are ranging over intensions, i.e, entities determining the extension of theoretical terms. This would result in a deviation from standard second order logic. Eventually, one should discuss whether intensions - in the sense of entities determining the extension of theoretical terms - are better viewed as individuals.

$5 \mathfrak{A}_{o}$ is thought of here as identical to the corresponding intended interpretation of the observational language in the Ramsey view. In discussions of this view, it is usually not made explicit whether or not this interpretation is such that it determines the truth-value of every $L\left(V_{o}\right)$ sentence, including all observable truths of the future world history. Our impression is that such an assumption is implicitly made. Alternatively, $\mathfrak{A}_{\mathfrak{o}}$ may be restricted to interpretations that determine the truth-values of $L\left(V_{o}\right)$ sentences concerning the observable world until the present time. Which decision is made seems, however, not essential in the context of the present paper. 
ii) $\nu(\phi):=F$ iff for every structure $\mathfrak{A} \in \mathbf{S}_{\mathfrak{a}}, \mathfrak{A}=\phi$ does not hold

iii) $\nu(\phi)$ is indeterminate iff there is at least one structure $\mathfrak{A}_{1}$ for which $\mathfrak{A}_{1}=\phi$ holds true and at least another structure $\mathfrak{A}_{2}$ for which $\mathfrak{A}_{2} \models \phi$ does not hold true, where both $\mathfrak{A}_{1}$ and $\mathfrak{A}_{2}$ are members of $\mathbf{S}_{\mathfrak{a}}$, the set of admissible structures.

The idea behind these rules is rather simple. A theoretical sentence is true iff it is true in every admissible structure. A theoretical sentence is false iff it is false in every admissible structure. And a sentence has no determinate truth-value iff it is true in - at least - one admissible structure and false in - at least - another structure being also admissible. Obviously, the truth-rules allow theoretical sentences to have determinate values even in the absence of a unique determination of theoretical terms by postulates. It should be noted further that the truth-value of a logically true or logically false theoretical sentence is not altered if definition 2 is adopted. As is obvious from part iii) of definition 2 , there are also theoretical sentences that cannot be assigned to a determinate truth-value. ${ }^{6}$

In a case where there is no structure that extends $\mathfrak{A}_{o}$ and satisfies the postulates $\Phi_{T C}$, it is not sensible to impose a constraint on the $L\left(V_{o}, V_{t}\right)$ interpretations by the postulates. Then, the intended interpretation of the theoretical terms collapses. There are several options on how to formally represent such a failure of interpretation. To consider every member of $\operatorname{EXT}\left(\mathfrak{A}_{o}, V_{t}, D_{t}\right)$ admissible is only one such option. This choice has the merit of not affecting the interpretation of the observational language. Another option to formally account for the case where the intended interpretation of theoretical terms collapses - because of $\operatorname{MOD}\left(\Phi_{p}\right) \cap \operatorname{EXT}\left(\mathfrak{A}_{o}, V_{t}, D_{t}\right)$ being empty - is to assign every theoretical sentence the value false. Such a choice has the disadvantage that the law of excluded middle would not hold for theoretical sentences. If we were to set every theoretical sentence to false in case of $\operatorname{MOD}\left(\Phi_{p}\right) \cap \operatorname{EXT}\left(\mathfrak{A}_{o}, V_{t}, D_{t}\right)$ being empty, then we would have to come up with two logically calculuses, one for the case where $\operatorname{MOD}\left(\Phi_{p}\right) \cap E X T\left(\mathfrak{A}_{o}, V_{t}, D_{t}\right)$ is empty, and another where it is not.

For the definition of the set $\mathbf{S}_{\mathfrak{a}}$ of admissible structures it has been assumed that the domain $D_{t}$, in which the theoretical terms are interpreted, does not vary. This assumption is justified in the framework of Carnap's dual-level conception of scientific language. For, there it is assumed that the individual constants

\footnotetext{
${ }^{6}$ These truth-rules are inspired by supervaluation logic and we are deeply indebted to Priest's (2001) exposition of this logic. In using supervaluations for a formal account of vagueness, the notion of supertruth has been introduced by Williamson (1994), p. 142-153, and others. This notion formally coincides with the notion of truth being defined by the above truth-rules. Elsewhere, we dealt with supervaluations and theoretical terms in the framework of truth-value-semantics in the sense of LeBlanc (1976). See Andreas (2008). This treatment is somewhat more tedious than the one given in the present paper.

As in certain versions of supervaluation logic, a set $\mathbf{S}_{\mathfrak{a}}$ of admissible structures may well verify a disjunction without verifying either disjunct, or verify an existential quantification without verifying any instance of it. This amounts to a deviation from what philosopher's have called a common sense view of the world. Likewise, the idea of representing a part of the world through a set of structures that has usually more than one member as opposed to representing it through a single structure is a deviation from common sense. The very nature of theoretical terms - more precisely, the non-uniqueness of their interpretation - requires such a deviation.
} 
of $V_{t}$ are mathematical symbols having a determinate interpretation. If $D_{t}$ is supposed to contain spatiotemporal theoretical entities, such as electrons, atoms, or molecules, it may justified to modify the definition of $\mathbf{S}_{\mathfrak{a}}$ such that a structure is admissible iff it extends $\mathfrak{A}_{o}$ and satisfies the postulates, where $V_{t}$ is interpreted in some domain $D_{t}$. Whether or not $D_{t}$ is considered constant is not essential to the modal view to be developed. Our preference for a constant domain of theoretical entities goes back to Carnap (1958)/(1975) but may well be discarded.

In a certain sense, the problem of how to deal with theoretical sentences whose terms are not properly interpreted is similar to the problem that arises in the case of sentences with proper names without referents, as in sentences about the present king of France. In both cases we are concerned with sentences in which at least one term occurs that cannot be interpreted in the intended way. Considering such sentences having no determinate truth-value is one option, considering them false another.

Even though the primary function of postulates is to interpret theoretical terms, the truth-value assignment to sentences that are postulates is not entirely void of empirical content. According to the truth-rules for theoretical sentences in definition 2, a sentence being a member of $\Phi_{T C}$ is true if $M O D\left(\Phi_{T C}\right) \cap$ $\operatorname{EXT}\left(\mathfrak{A}_{o}, V_{t}, D_{t}\right)$ is not empty. Further, it holds that every member of $\Phi_{T C}$ is assigned to the value true iff there is a structure extending $\mathfrak{A}_{o}$ and satisfying the postulates $\Phi_{T C}$. Whether or not there is such a structure also depends on $\mathfrak{A}_{o}$, and hence on empirical facts. The criteria according to which the epistemic quality of a system of postulates can be judged may be summarized by the following proposition:

Proposition 1. A theory may be given as a set of postulates $\Phi_{T C}$. As rules for the truth-value assignment to theoretical sentences the rules in definition 2 are adopted. Then, the following criteria are equivalent:

i) Every $\phi \in \Phi_{T C}$ is assigned to the value true

ii) $\operatorname{MOD}\left(\Phi_{T C}\right) \cap \operatorname{EXT}\left(\mathfrak{A}_{o}, V_{t}, D_{t}\right)$ is not empty

iii) $T C^{R}$ is true.

Equivalence between i) and ii): For the left-to-right direction, assume that every $\phi \in \Phi_{T C}$ is assigned to the value true. As assumption of the indirect proof, suppose $\operatorname{MOD}\left(\Phi_{T C}\right) \cap \operatorname{EXT}\left(\mathfrak{A}_{o}, V_{t}, D_{t}\right)$ is empty so that $\mathbf{S}_{\mathfrak{a}}=\operatorname{EXT}\left(\mathfrak{A}_{o}\right.$, $\left.V_{t}, D_{t}\right)$. Let $\mathfrak{A}_{1}$ be any member of $\mathbf{S}_{\mathfrak{a}}$. Then, we can infer from $\nu(\phi)=\mathrm{T}$ for every $\phi \in \Phi_{T C}$ and definition 2 that $\mathfrak{A}_{1} \models \phi$ for every $\phi \in \Phi_{T C}$. Hence, $\mathfrak{A}_{1} \in \operatorname{MOD}\left(\Phi_{T C}\right)$. Because of this and $\mathfrak{A}_{1} \in \operatorname{EXT}\left(\mathfrak{A}_{o}, V_{t}, D_{t}\right), \operatorname{MOD}\left(\Phi_{T C}\right) \cap$ $\operatorname{EXT}\left(\mathfrak{A}_{o}, V_{t}, D_{t}\right)$ is not empty. Contradiction. The right-to-left direction of the proof is trivial.

Equivalence between ii) and iii): For the left-to-right direction, assume that $\operatorname{MOD}\left(\Phi_{T C}\right) \cap \operatorname{EXT}\left(\mathfrak{A}_{o}, V_{t}, D_{t}\right)$ is not empty. Then, there is a structure $\mathfrak{A}=\left(D_{o}, D_{t}, \mathfrak{a}_{o}, \mathfrak{a}_{t}\right)$ such that $\mathfrak{A}=\Phi_{T C}$, where $\mathfrak{A}_{0}=\left(D_{o}, \mathfrak{a}_{o}\right)$ is the intended interpretation of $L\left(V_{o}\right)$. Then, obviously, there is an assignment to the second order variables in the open formula $T C\left(n_{1}, \ldots, n_{k}, X_{1}, \ldots, X_{n}\right)$ such that this 
formula is assigned to the value true, where the $V_{o}$ symbols are interpreted according to $\mathfrak{A}_{o}$. (Simply take the interpretation of the $V_{t}$ terms, as given by the structure $\mathfrak{A}$, as assignment to the second order variables using the correspondence between these variables and $V_{t}$ terms.) Hence, $\left(D_{o}, D_{t}, \mathfrak{a}_{o}\right) \models(T C)^{R}$. For the right-to-left direction, assume $\left(D_{o}, D_{t}, \mathfrak{a}_{o}\right) \models(T C)^{R}$, where $\mathfrak{A}_{o}=\left(D_{o}, \mathfrak{a}_{o}\right)$ is the intended interpretation of $L\left(V_{o}\right)$. Then, there is an assignment of values to the second order variables of the open formula $T C\left(n_{1}, \ldots, n_{k}, X_{1}, \ldots, X_{n}\right)$ such that this formula is assigned to the value true. If this assignment is taken as an interpretation of the $V_{t}$ terms using the correspondence between the variables of $(T C)^{R}$ and theoretical terms, while the $V_{o}$ terms are interpreted according to $\mathfrak{A}_{o}$, then every sentence of $\Phi_{T C}$ comes out true under this interpretation. Call the functions representing this interpretation of the $V_{t}$ terms $\mathfrak{a}_{t 1}$. Then, $\left(D_{o}, D_{t}, \mathfrak{a}_{o}, \mathfrak{a}_{t 1}\right)$ is an extension of the intended interpretation of $L\left(V_{o}\right)$ and also satisfying the postulates $\Phi_{T C}$. Hence, $\operatorname{MOD}\left(\Phi_{T C}\right) \cap \operatorname{EXT}\left(\mathfrak{A}_{o}, V_{t}, D_{t}\right)$ is not empty.

Why would it not be correct in general to claim that $\phi \in \Phi_{T C}$ is true iff $\operatorname{MOD}\left(\Phi_{T C}\right) \cap \operatorname{EXT}\left(\mathfrak{A}_{o}, V_{t}, D_{t}\right)$ is not empty? This is because in the case of a C-axiom, that is, an axiom having occurrences of both $V_{t}$ and $V_{o}$ symbols, it may happen that this axiom holds true in every $\mathfrak{A} \in \operatorname{EXT}\left(\mathfrak{A}_{o}, V_{t}, D_{t}\right)$.

Why did we come to label the present account of the semantics of theoretical sentences as a modal view? Now, the truth-rules of definition 2, obviously, show a striking similarity to the rules for the box operator in simple modal systems in which the accessibility relation is an equivalence relation and every world is accessible from any other. Such a system is based on the idea that a proposition is necessarily true iff it is true in every possible world. In a likewise manner, a theoretical sentence is assigned to the value true iff this sentence is true in every admissible structure. An explicit modal notation for this view will be given in the next section.

It seems worth noting that Mikenberg et al. (1986) and Da Costa and French (1990) introduced a notion of pragmatic truth whose definition resembles the model-theoretic restatement of the Ramsey sentence given by clause ii) of proposition 1 . More precisely, a sentence $\phi$ is said to be pragmatically true in a structure $\mathfrak{A}$ (relative to a set $P$ of sentences) if there exists a total extension $\mathfrak{B}$ of $\mathfrak{A}$ such that $\mathfrak{B}=\phi$ and, for every $\psi \in P, \mathfrak{B} \models \psi$. If $\phi$ is not pragmatically true in $\mathfrak{A}$, it is said to be pragmatically false in $\mathfrak{A}$ relative to $P$ (Mikenberg et al. (1986), p. 204). $P$ is a set of primary statements which need not be observational but whose truth must be understood in the correspondence sense. If the concept of pragmatic truth is only applied to whole theories, which seems to be the intention of Da Costa and French (1990), p. 257, then it is equivalent to the model-theoretic restatement of the Ramsey sentence, with the qualification that a set $P$ of primary statements needs to be satisfied by an extension of $\mathfrak{A}$ satisfying the theory. If the pragmatic conception of truth is also applied to scientific statements other than axioms of a theory, which seems to be intended by Mikenberg et al. (1986), p. 204, it leads to plain contradictions. For, there may well be an extension of $\mathfrak{A}$ satisfying $P$ in which $\phi$ is true and another one also satisfying $P$ in which $\neg \phi$ is true as well. Then, both $\phi$ and $\neg \phi$ would have to 
be considered pragmatically true in $\mathfrak{A}$ relative to $P$. This type of contradiction has been circumvented in the modal view.

\section{Asserting Theoretical Sentences}

We are now in a position to say how the assertion of a theoretical sentence can be represented in a model-theoretic fashion. For this purpose, we propose to adopt the following notation for the assertion of any $L\left(V_{o}, V_{t}\right)$ sentence:

$$
\text { (2) } \quad \mathbf{S}_{\mathfrak{a}}={ }_{m} \phi \text {. }
$$

In words: For all structures $\mathfrak{A} \in \mathbf{S}_{\mathfrak{a}}$ it holds that $\mathfrak{A} \models \phi$, where $\mathbf{S}_{\mathfrak{a}}$, the set of admissible structures, is defined by definition 1 . This notation is primarily intended to represent the assertion of those theoretical sentences that are not postulates. Even so, it does also represent the assignment of truth-values to sentences that are postulates, provided the truth-rules in definition 2 are adopted for such sentences. Moreover, the notation applies to observational sentences because, for all sentences $\phi$ of $L\left(V_{o}\right), \mathbf{S}_{\mathfrak{a}} \models_{m} \phi$ iff $\mathfrak{A}_{o} \models \phi$, where $\mathfrak{A}_{o}$ is the intended interpretation of the observational language.

In having given an explicit, semantically well founded notation for asserting theoretical sentences, the major goal of the present paper has been achieved. This goal is motivated by the difficulties arising in the Ramsey view when it comes to representing the meaning of a theoretical sentence. Ramsey's proposal to take such a sentence $\phi$ to mean the difference between $\left(T C \wedge \phi_{a} \wedge \phi\right)^{R}$ and $\left(T C \wedge \phi_{a}\right)^{R}$ was found cumbersome and, more importantly, not sufficiently formal for our use. These shortcomings of the Ramsey view appear to have overcome in the modal view expounded. As just shown, a formal notation for the semantics of theoretical sentences is available there. At the same time, is the modal view capable of retaining those features of the Ramsey view which were considered merits. This may be shown by comparing notation (2) with the standard notation for the assertion of a sentence $\phi$ in the framework of model-theory, which goes as follows:

$$
\text { (3) } \quad \mathfrak{A}_{\mathfrak{i}}=\phi
$$

where $\mathfrak{A}_{\mathfrak{i}}$ stands for the intended interpretation of the language. ${ }^{7}$

\footnotetext{
${ }^{7}$ Admittedly, model-theory by itself makes no notation for asserting a sentence available. Unlike Frege's Begriffsschrift, model-theory does not contain an assertion sign. For this reason, model-theoretic semantics is best viewed as interpretational semantics in the sense that it tells us what it is for a sentence to be true only with respect to a particular interpretation of the non-logical symbols of the language and the specification of the universe in which the variables are interpreted. This view has been articulated, among others, by Etchemendy (1999). Likewise, Carnap has pointed out that the model-theoretic apparatus of predicate logic is apt to clarify the notions of logical truth and logical consequence but makes no contribution
} 
In the standard notation, a compositional account of sentence meaning is assumed which leaves no room for contextual aspects of meaning. This contrasts with the notation (2) in the modal view, where the semantic value of a theoretical sentence essentially depends on the theoretical context. For, according to notation (2), a theoretical sentence is true iff it is true in every admissible structure. And the range of admissible structures is not only determined by the intended interpretation of the observational language but also by the postulates $\Phi_{T C}$. Hence, the meaning of a theoretical sentence is, to some extent, determined by the axioms of a scientific theory. These axioms can thus be seen as giving the theoretical context in which theoretical sentences are being asserted. Underlying this consideration is a notion of meaning being equated with Frege's notion of sense. That is, the sense of an expression is what determines its extension. Thus, the sense of a sentence is what determines its truth-value.

It can furthermore be shown that the problem of theoretical terms does not arise in the modal view. In this view, the truth-value of a postulate is not seen as being determined by the extension of the theoretical terms. The assignment of truth-values to postulates is rather part of the interpretation of theoretical terms. Thus, there is, arguably, another solution to the problem of theoretical terms, in addition to the one given by the Ramsey sentence. For a closer examination of this point see again Andreas (2007).

The present notation for asserting theoretical sentences can be seen to differ in two major respects from what has been taken as the standard notation for such assertions. First, sentences of the object language are allowed to determine which interpretations of the language are the intended ones. Second, in place of a single intended interpretation there is now a range of interpretations of which each single interpretation equally qualifies as being intended. The latter feature will be exploited in the following attempt to give an explicit modal notation for the assertion of a theoretical sentence.

A structure for a modal first order language being two-sorted in the manner described is a sextuple of the following type:

(4) $\mathfrak{M}=\left\langle W, R, D_{o}, D_{t},\left\{V_{o, w}\right\}_{w \in W},\left\{V_{t}\right\}_{w \in W}\right\rangle$

where $W$ is a set of states, $R$ an accessibility relation, $\left\{V_{o, w}\right\}_{w \in W}$ a valuation function for the $V_{o}$ symbols, and $\left\{V_{t, w}\right\}_{w \in W}$ is such a function for predicates and individual constants of $V_{t}$. (For reasons of simplicity it is assumed that $\mathrm{n}$-place function symbols are represented as $(\mathrm{n}+1)$-place predicates.) These

to an understanding of the notion of factual truth. See Carnap (1973), p. 98n.

To do justice to our plain and representational understanding of truth within the framework of model-theoretic semantics, the notion of an intended interpretation has been invoked sensibly. (See, e.g., Carnap (1973), p. 101.) An intended interpretation of a formal system represents the meaning of the non-logical symbols. It can be made explicit by so-called rules of designation which assign either an intensional or an extensional interpretation to these symbols by means of expressions of a meta-language, where every intensional interpretation uniquely determines an extensional one. Of course, the domain in which the variables are interpreted must also be specified. 
valuation functions are simply assigning an interpretation to the non-logical symbols of $L\left(V_{o}, V_{t}\right)$ for every state being a member of $W$. It is assumed that the domains of interpretation, $D_{o}$ and $D_{t}$, do not vary among the different states. This assumption holds for the intended interpretations of $L\left(V_{o}, V_{t}\right)$, as has been made explicit above.

How can we introduce a set $W$ of states and the accessibility relation $R$ in such a manner that the resulting modal logic provides us with a means to represent the semantics of theoretical sentences? In light of what has been said in the last section, this question is not difficult to answer. The following definitions may be adopted:

Definition 3. $W:=\mathbf{S}_{\mathfrak{a}}$, where $\mathbf{S}_{\mathfrak{a}}$ is the set of admissible structures in the sense of definition 1.

Definition 4. $R:=\{(x, y) \mid x \in W$ and $y \in W\}$.

Importantly, $R$ is an equivalence relation such that every member of $W$ is accessible from any other one, and also from itself, which holds independently of which $L\left(V_{o}, V_{t}\right)$ structures are admissible. In such kind of modal structures, the valuation of a formula with a box operator and the valuation of a formula with a diamond operator do not depend on the particular state. The semantics of the modal box and the modal diamond operator can therefore be simplified as follows:

(5) $\mathfrak{M}, g \models_{\mathfrak{m}} \quad$ iff for all $v \in W$ we have $\mathfrak{M}, g, v \models_{\mathfrak{m}} \phi$

(6) $\mathfrak{M}, g \models_{\mathfrak{m}} \diamond \phi$ iff for some $v \in W$ we have $\mathfrak{M}, g, v \models_{\mathfrak{m}} \phi$

where $g$ is a (two-sorted) assignment function for variables and $\phi$ a formula of the language. If $\phi$ is a sentence, then $g$ drops out as inessential.

The following proposition is now almost trivial:

Proposition 2. For all $L\left(V_{o}, V_{t}\right)$ sentences $\phi: \mathfrak{M} \models_{\mathfrak{m}} \square \phi$ iff $\mathbf{S}_{\mathfrak{a}} \models_{m} \phi$,

where $\mathfrak{M}=_{\mathfrak{m}} \square \phi$ stands for the claim that the modal first order sentence $\square \phi$ is true in the modal structure $\mathfrak{M}$. It can be proved by applying the rule for the semantics of the box operator.

Thus, we have obtained another model-theoretic representation of the claim that a theoretical sentence $\phi$ is true:

$$
\text { (7) } \mathfrak{M} \models_{\mathfrak{m}} \square \phi
$$

where $\mathfrak{M}$ is introduced as just described. Moreover, we can say that a theoretical sentence $\phi$ is indeterminate in the sense of definition 2 iff $\mathfrak{M} \models_{\mathfrak{m}} \diamond \neg \phi \wedge \diamond \phi$. 
So there are two notations for the assertion of a theoretical sentence in the modal view, one of them being explicitly modal, the other being modal only in spirit. Both notations give a representation of sentence meaning for theoretical sentences and therefore solve a major difficulty of the Ramsey view.

The notion of truth encoded by notation (7) bears a certain resemblance with the notion of T-necessity, according to which $\phi$ is T-necessary iff it is logically implied by the theory T. (See, for example, van Fraassen (1980), p. 201-203.) Yet these notions are not equivalent. Whereas $\mathfrak{M} \models_{\mathfrak{m}} \square \phi$ holds for every T-necessary sentence, provided the axioms of $T$ are taken as the postulates $\Phi_{T C}$ and $\operatorname{MOD}\left(\Phi_{T C}\right) \cap \operatorname{EXT}\left(\mathfrak{A}_{o}, V_{t}, D_{t}\right)$ is not empty, it does not follow from $\mathfrak{M} \models_{\mathfrak{m}} \square \phi$ that $\phi$ is T-necessary. Further, the intent of notation (7) is to explicate ordinary truth of scientific statements and not a kind of necessary truth being independent of empirical facts.

\section{Deductive Reasoning}

A second difficulty that was found in section 2 to inhere the Ramsey view concerns deductive reasoning. If a theoretical sentence $\phi$ is translated to $(T C \wedge$ $\left.\phi_{a} \wedge \phi\right)^{R}$ in this view, then the resulting notation can hardly be used for drawing inferences from $\phi$. It is therefore difficult to see how the Ramsey view may account for inferential practice in science. There, theoretical sentences are used in deductions without any substitutions of the theoretical terms by second order variables in the Ramsey style. If a theoretical sentence is taken to mean the difference between $\left(T C \wedge \phi_{a} \wedge \phi\right)^{R}$ and $\left(T C \wedge \phi_{a}\right)^{R}$, then the resulting notation is not sufficiently formal for being used in deductions. Do we have to resort, for these reasons, to the standard view when an account of deductive reasoning in science is called for? This is not necessary. Rather, we will show that the modal view is also capable of giving such an account, provided non-monotonic aspects of scientific reasoning are set aside and classical first order logic is considered a proper explication of deductions in science.

Two notations were given for the assertion of a theoretical sentence in the modal view: i) $\mathbf{S}_{\mathfrak{a}} \models_{m} \phi$, and ii) $\mathfrak{M} \models_{\mathfrak{m}} \square \phi$. The first notation does - with respect to the right side of the symbol of the satisfaction relation - not differ from the standard view, in which the assertion of $\phi$ is represented by $\mathfrak{A}_{\mathfrak{i}}=\phi$, where $\mathfrak{A}_{\mathfrak{i}}$ is the intended interpretation of the language. Let us adopt any logical first order calculus being complete and sound with respect to standard first order semantics as the logical part of the deductive system corresponding to notation i) of the modal view. For notation ii), which is explicitly modal, the modal system S5 suggests itself as logical part of the deductive system.

Let us assume that classical first order logic is apt to account for inferential practice in science. Then, we can say that the modal view in the form of notation i) $\left(\mathbf{S}_{\mathfrak{a}} \models_{m} \phi\right)$ accords with inferential practice in science if the adoption of a classical deductive system can be justified for deductions with propositions in this notation. Now, in classical logic, a proof of soundness and completeness of the deductive system is considered a justification of this system, provided 
truth-conditional semantics is adopted. What we have to show therefore is that the logical part of a classical deductive system is also complete and sound with respect to the peculiar relation of semantic consequence we are concerned with in the modal view. In the modal view, we have a preference for inferences that are truth-preserving in the sense that if the premisses are true in a set of structures, then the conclusion is also true in this set of structures. Let us be a bit more explicit about semantic consequences in the modal view:

Definition 5. A sentence $\phi$ is a semantic consequence of a set $\Phi$ of sentences in the modal view - in symbols: $\Phi \models_{m} \phi$ - iff it holds that $\mathbf{S}_{\mathfrak{a}} \models_{m} \phi$ whenever $\mathbf{S}_{\mathfrak{a}} \models_{m} \Phi$ for any set $\mathbf{S}_{\mathfrak{a}}$ of admissible structures in the sense of definition 1 .

Proposition 3. For all sets $\Phi$ of $L\left(V_{o}, V_{t}\right)$ sentences and all sentences $\phi$ of $L\left(V_{o}, V_{t}\right): \Phi \models{ }_{m} \phi$ iff $\Phi \models \phi$.

Proof. For the left-to-right direction, assume that $\Phi=_{m} \phi$, where the members of $\Phi$ and $\phi$ itself are $L\left(V_{o}, V_{t}\right)$ sentences. As assumption of the indirect proof, suppose that $\Phi=\phi$ does not hold, i. e., $\phi$ is not a classical semantic consequence of $\Phi$. Then, there is a structure $\mathfrak{A}_{1}=\left(D_{o 1}, D_{t 1}, \mathfrak{a}_{o 1}, \mathfrak{a}_{t 1}\right)$ such that $\mathfrak{A}_{1} \models \Phi$ but not $\mathfrak{A}_{1} \models \phi$. Now, we need to construct a set $\mathbf{S}_{\mathfrak{a} 1}$ of structures in the sense of definition 1 such that $\mathfrak{A}_{1} \in \mathbf{S}_{\mathfrak{a} 1}$. For this, take $\mathfrak{A}_{o 1}=\left(D_{o 1}, \mathfrak{a}_{o 1}\right)$ as intended interpretation of $L\left(V_{o}\right)$. Then, let $\Phi_{1}$ be the set of sentences that are members of $\Phi$ and have occurrences of theoretical terms. Further, take $\Phi_{1}$ as the set of postulates for defining the set of admissible structures. Then, we obtain $\mathbf{S}_{\mathfrak{a} 1}=$ $\operatorname{MOD}\left(\Phi_{1}\right) \cap \operatorname{EXT}\left(\mathfrak{A}_{o 1}, V_{t}, D_{t}\right)$. Since $\mathfrak{A}_{1} \in \operatorname{MOD}\left(\Phi_{1}\right)$ and $\mathfrak{A}_{1} \in \operatorname{EXT}\left(\mathfrak{A}_{o 1}, V_{t}\right.$, $\left.D_{t}\right)$, it holds that $\mathfrak{A}_{1} \in \mathbf{S}_{\mathfrak{a} 1}$. By assumption we know that $\Phi=_{m} \phi$. From this, $\mathfrak{A}_{1} \in \mathbf{S}_{\mathfrak{a} 1}$, and $\mathbf{S}_{\mathfrak{a} 1} \models_{m} \Phi$, we can infer that $\mathfrak{A}_{1} \models \phi$. Contradiction.

For the right-to-left direction, assume that $\Phi=\phi$. As assumption of an indirect proof, suppose that $\Phi=_{m} \phi$ does not hold. So, there is a set $\mathbf{S}_{\mathfrak{a} 1}$ of structures such that $\mathbf{S}_{\mathfrak{a} 1} \models_{m} \Phi$ but not $\mathbf{S}_{\mathfrak{a} 1} \models_{m} \phi$. Hence, there is at least one structure $\mathfrak{A}_{1} \in \mathbf{S}_{\mathfrak{a} 1}$ such that $\mathfrak{A}_{1} \models \Phi$ but not $\mathfrak{A}_{1} \models \phi$. Therefore, $\Phi \models \phi$ does not hold. Contradiction.

By proposition 3 we know that once a classical logical calculus being sound and complete with respect to classical first order semantics is adopted for notation i) of the modal view, the resulting deductive system is sound and complete with respect to the semantics of the modal view. This justifies the adoption of a classical logical calculus. Hence, we can say that the modal view in the form of notation i) accords with inferential practice in science to the extent in which the standard view accords with this practice. This seems like an important advantage that the modal view has to the Ramsey view.

Let us now move to the explicit modal notation of the modal view. Admittedly, this notation does not accord with inferential practice in science because modal operators are used in science only very rarely. At least, ordinary scientific statements are - contrary to the suggestion of the modal view in the form of notation ii) - not prefixed by a modal operator. It seems feasible, however, to recognize a relation between deductions in the modal view and deductions in a classical deductive system in view of the following proposition: 
Proposition 4. For all finite sets $\Phi$ of first order sentences not containing modal operators and all first order sentences $\phi$ not containing such an operator: $\square \Phi \vdash_{S 5} \square \phi$ iff $\Phi \vdash \phi$.

$\vdash_{S 5}$ designates derivability in the modal system S5 and $\vdash$ derivability in a complete and sound axiomatization of first order logic. A proof can be obtained using deduction theorem.

By this proposition we know that if we were to adopt the modal view in the form of the modal notation for making assertions and drawing inferences in science, we would obtain a relation of derivability that is equivalent to derivability in the standard view. Such a result was not obtained for the Ramsey view.

The purpose of the modal view is merely to gain a semantic insight into the nature of human theorizing. This view can be seen as a semantic adjustment of the standard view, which leaves the deductions corresponding to the standard view intact.

\section{A Note on Competing Theories}

In a certain sense, the modal view elucidates a theory-immanent notion of truth. Consider two theories, $T_{1}$ and $T_{2}$, such that each is compatible with the observational facts, but from $T_{1} \cup T_{2}$ a contradiction can be derived. (These conditions are satisfied by empirically equivalent and yet logically incompatible theories.) In the modal view, we would then be able to introduce two modal operators, $\square_{1}$ and $\square_{2}$, where $W_{i}=\operatorname{MOD}\left(\Phi_{T_{i}}\right) \cap \operatorname{EXT}\left(\mathfrak{A}_{o}, V_{t i}\right.$, $\left.D_{t i}\right), \mathrm{i}=1,2$. The semantics of these operators is based on modal structures of the type $\mathfrak{M}=\left\langle W_{1}, W_{2}, R_{1}, R_{2}, D_{o}, D_{t 1}, D_{t 2},\left\{V_{o 1, w}\right\}_{w \in W_{1}},\left\{V_{o 2, w}\right\}_{w \in W_{2}}\right.$, $\left.\left\{V_{t 1}\right\}_{w \in W_{1}},\left\{V_{t 2}\right\}_{w \in W_{2}}\right\rangle$ in a manner being analogous to definition 5. If, then, from $T_{1}$ a sentence $\phi_{1}$ can be derived, while $\neg \phi_{1}$ is derivable from $T_{2}$, this implies that $\mathfrak{M} \models_{\mathfrak{m}} \square_{1} \phi_{1}$ and $\mathfrak{M} \models_{\mathfrak{m}} \square_{2} \neg \phi_{1}$, provided $\operatorname{MOD}\left(\Phi_{T_{i}}\right) \cap \operatorname{EXT}\left(\mathfrak{A}_{o}, V_{t i}\right.$, $\left.D_{t i}\right), \mathrm{i}=1,2$, is not empty. There is then no danger of inconsistent assignments of semantic values to one and the same sentence. Nor becomes the deductive system contradictory in this case, even though both theories are formulated in one and the same language.

The admission of different, not necessarily converging conceptions of truth at the theoretical level within the modal view seems to accord with certain antirealist intuitions about theoretical truth and will also appeal to advocates of a historically relativized notion of truth. But such a liberal view of truth at the theoretical level may equally well serve as an objection to the modal view, particularly since this view provides - in its present exposition - no means to account for a non-theory-immanent notion of truth, according to which competing theories have the same claim to truth. To this objection we reply that the modal view may enable us to discover new implications of the positions that are involved in the debate about realism and antirealism. Notably, the kind of antirealism that may eventually be based upon the modal view differs from the kind of antirealism that Dummett (1991) advocated since the modal view does - 
unlike intuitionistic logic - not require a revision of inferential practice in science. Another move available as a reply to the objection is to dismiss the proposal of allowing the introduction of different modal box operators through which different, not necessarily converging concepts of truth are admitted. Then, both of two contradictory theories would be a member of $\Phi_{T C}$ and $\operatorname{MOD}\left(\Phi_{T C}\right) \cap$ $\operatorname{EXT}\left(\mathfrak{A}_{o}, V_{t}, D_{t}\right)$ would be empty. But the latter reply seems less congenial to the spirit of the modal view.

If the modal view is rejected because of a prior preference for a more absolute notion of truth, one is left with no minor challenge. One would then have to show that it is not sensible to explicate the semantics of scientific expressions along the lines of theoretical terms. Alternatively, one may try to show that the determination of theoretical terms through the axioms of a scientific theory is such that the extension of these terms is uniquely determined.

\section{Conclusion}

A major difficulty in formally representing the semantics of theoretical sentences arises from there not being a unique determination of theoretical terms through postulates, which are supposed to interpret these terms. Aside from special cases, there is a range of interpretations of the theoretical language $L\left(V_{o}, V_{t}\right)$ that extend a given interpretation of the observational language $L\left(V_{o}\right)$ and satisfy the postulates. In the Ramsey view this problem does not arise because the Ramsey sentence implies no assertion about the uniqueness of interpretation of the theoretical terms. But we have seen that the Ramsey view suffers from another difficulty, namely the formal representation of the assertion of a theoretical sentence. For this reason, deductive reasoning with theoretical sentences can hardly be represented in the Ramsey view. These two, interrelated difficulties are overcome by the modal view expounded in the present paper.

The basic idea of the modal view is to introduce a notion of truth according to which a sentence is true iff it is true in every admissible structure. The set of admissible structures is defined as the set of $L\left(V_{o}, V_{t}\right)$ structures extending a given $L\left(V_{o}\right)$ structure and satisfying the postulates, provided there is any structure meeting these conditions. This notion of truth allows theoretical sentences to have determinate values, even in the absence of a unique interpretation of theoretical terms by postulates. The introduction of an explicit modal notation for the assertion of a theoretical sentence is then straight forward.

What we have been arguing for is that scientific statements are better understood as propositions of the form $\mathfrak{M} \models_{\mathfrak{m}} \square \phi$ than as propositions of the form $\mathfrak{A}=\phi$, where $\mathfrak{M}$ encompasses a range of intended interpretations, while $\mathfrak{A}$ designates a single intended interpretation. Hence, the divergence of the modal view from standard semantics consists in rejecting the assumption of a unique intended interpretation of the language in which a scientific theory is couched. The justification of this divergence is again straight forward in light of the above considerations: Once we are convinced that there are theoretical terms, i. e., non-logical symbols that get their meaning determined through the axioms 
of a scientific theory, the assumption of a unique intended interpretation being essential to standard semantics is not tenable any more.

\section{References}

Andreas, H. (2007). Carnaps Wissenschaftslogik. mentis, Paderborn.

Andreas, H. (2008). Another Solution to the Problem of Theoretical Terms. Erkenntnis 69: 315-333.

Braithwaite, R. B. (1955). Scientific Explanation. Cambridge University Press, Cambridge.

Carnap, R. (1973). Introduction to Symbolic Logic and its Applications. Dover Publications, New York.

Da Costa, N. C. A. and French, S. (1990). The Model-Theoretic Approach in the Philosophy of Science. Philosophy of Science 57(2): 248-265.

Dummett, M. (1991). The Logical Basis of Metaphysics. Harvard University Press, Cambridge, Mass. 1991.

Etchemendy, J. (1999). The Concept of Logical Consequence. CSLI Publications, Stanford.

Feyerabend, P. K. (1962). Explanation, Reduction, and Empiricism. In: H. Feigl and G. Maxwell (eds.) Scientific Explanation, Space, and Time, University of Minnesota Press, Minneapolis, vol. 3 of Minnesota Studies in the Philosophy of Science, 28-97.

Ketland, J. (2004). Empirical Adequacy and Ramsification. British Journal for Philosophy of Science 55: 287-300.

LeBlanc, H. (1976). Truth-Value Semantics. North Holland Publishing Company, Amsterdam.

Mikenberg, I., Da Costa, N. C. A., and Chuaqui, R. (1986). Pragmatic Truth and Approximation to Truth. Journal of Symbolic Logic 51(1): 201-221.

Quine, W. v. O. (1961). Two Dogmas of Empiricism. In: From a Logical Point of View, Harvard University Press, Cambridge, Mass., 2nd edn., 20-46.

Ramsey, F. P. (1950). Theories. In: R. B. Braithwaite (ed.) The Foundations of Mathematics and Other Logical Essays, Humanities Press, New York, 212236 .

Schurz, G. (2005). Semantic Holism and (Non-)Compositionality of Scientific Theories. In: M. Werning, E. Machery, and G. Schurz (eds.) The Compositionality of Meaning and Content. Vol. I, Ontos-Verlag, Frankfurt, 271-284.

Sneed, J. (1979). The Logical Structure of Mathematical Physics. D. Reidel Publishing Company, Dordrecht, 2nd edn. 
van Benthem, J. (1978). Ramsey Eliminability. Studia logica 37: 321-336.

van Fraassen, B. (1980). The Scientific Image. Clarendon Press, Oxford. 